\title{
APPLYING DATA MINING TO SOFTWARE DEVELOPMENT PROJECTS: A CASE STUDY
}

\author{
Jacinto Mata, José Luis Álvarez \\ Dpto. Ingeniería Electrónica, Sistemas Informáticos y Automática \\ Universidad de Huelva, Spain \\ José Cristóbal Riquelme, Isabel Ramos \\ Dpto. Lenguaje y Sistemas Informáticos \\ Universidad de Sevilla, Spain
}

Keywords: Software Development Project, Knowledge Discovery in Databases, Association Rules.

Abstract: One of the main challenges that the project managers have during the building process of a software development project (SDP) is to optimise the values of the parameters that measure the viability of the final process. The accomplishment of this task, something that was not easy at the beginning, was helped with the appearance of dynamic models and simulation environments. The application of data mining techniques to the managing of Software Development Projects (SDP) is not an uncommon phenomenon, as in any other productive process that generates information in the way of input data and output variables. In this paper, we present and analyze the results obtained from a tool, developed by the authors, based on a Knowledge Discovery in Databases (KDD) technique. One of the most important contributions of these techniques to the software engineering field is the possibility of improving the management process of an SDP. The purpose is to provide accurate decision rules in order to help the project manager to take decisions during the development.

\section{INTRODUCTION}

Since the development of software projects began on a great scale, one of the main concerns for the managers has been to optimize the attributes that measure the viability of the final process. At the beginning, such task was not easy to realize, since the manager himself was who, basing on his own experience, estimated the values where the attributes (management policies) that affect the development process had to move. The main problem was the amount of attributes that had to be estimated, since a wrong estimation in some of them could provoke that the final result did not fulfil the expectations.

A quite significant advance in the management of Software Development Projects (SDP) was achieved thanks to the appearance of dynamic models (AbdelHamid and Madnick, 1991), since they model the process carried out in the management of such projects. So, by means of the simulation, the manager can adjusts the attributes or input parameters in order that the output variables, delivery time, cost and quality, take those values that are considered as optimum for the process and final product.

The application of data mining techniques to the software engineering field is not very much spread.
Nevertheless, these techniques offer great advantages to managers of SDP. One of the most important contributions of these techniques to the software engineering field is the possibility of improving the management of the SDP process. The aim of data mining techniques is to provide the SDP manager knowledge to estimate which are the most suitable values of the attributes that influence in the project to optimize the values that estimate the viability of the project.

In this paper we try to contribute to the software engineering field applying a data mining technique to a set of software development project databases generated by a project simulator based in a dynamic model. We have used the algorithm GAR (Genetic Association Rule) to carry out our work because of its efficiency to discover association rules (Agrawal et al., 1993) in databases with attributes both discrete and continuous. Our final goal is to provide the expert with the knowledge induced from our databases, in this case in the way of association rules, which show the different associations that are produced among the input values in order that the output variables can be considered as goods. For example, we can obtain rules like: 


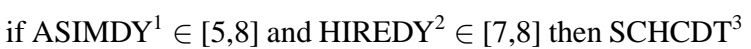
$\in[349,384]$ and ANERPT $^{4} \in[0.23,0.35]$

( support $=65 \%$, confidence $=100 \%)$

This rule shows that $65 \%$ of the projects that have been developed and have obtained a result considered by the expert as good for time and quality, have moved within values, as the ones shows in the rule for the attributes ASIMDY and HIREDY. In this case, the expert will be able to take into account this information to decide among which values must he to estimate the attributes ASIMDY and HIREDY so that the project can be considered as good.

The remainder of the paper is organized as follows: In Section 2, we present a brief description about the software engineering and dynamic systems and, in Section 3, we present how data mining techniques can be applied in software development projects. In Section 4 we explain which are the adaptations we have to do in order that the tool behave according to the expectations of the experts. We present the results of our experiments in Section 5. Finally, we offer concluding remarks in Section 7.

\section{SOFTWARE ENGINEERING AND DYNAMIC SYSTEMS}

As mentioned earlier, the use of dynamic models for SDP together with strong simulation environment (Stella, Vensim, iThink, PowerSim, etc.), has been of great help for the management of SDP (Chichakly, 1993).

A software project simulator allows to realise the following analysis: an a priori analysis of the project, project monitoring and post-mortem analysis. There is an important problem when using these simulators. In the software development process there are many parameters that affect the final result. In this way, the project manager must carry out numerous simulations and test with different values of the parameters that affect the process. The use of data mining reduces in part this problem and provides an alternative to help the SDP managers in the decision-taking in order to carry out a project with success.

\footnotetext{
${ }^{1}$ Average assimilation (of new personnel) delay (days)

${ }^{2}$ Hiring (of new personnel) delay (days)

${ }^{3}$ Delivery time (days)

${ }^{4}$ Quality (average number of errors per task)
}

\section{DATA MINING APPLICATION IN SDP}

Dynamic models for SDP include a set of attributes that allow us to define the management policies that can be applied in such projects, both those related with the project environment and those related with the development organization and the maturity level of the organization.

Once the attributes of the model are defined, the project manager must decide which are the variables that are going to be analyzed. The usual options are the variables that define the project development: delivery time, cost, number of mistakes generated, number of mistakes corrected, average development productivity, etc. So, the management policies that the mining process can find will be those that relate attribute values with the variables over whose influence we want to establish rules. Data mining bases its functioning on the withdrawing of information from databases. So, a database with information about already realized SDP would be necessary to carry out the mining process. The main problem is the nonexistence of these databases, so we will have to generate a database of projects realized with different values in the influential parameters by means of the simulators.

To generate a set of training cases for the mining process, the project manager must choose a range of values for the attributes of the model over which there is some uncertainty level. For example, the project manager does not know initially, if the average dedication of the technicians will be of $72 \%$ or $50 \%$, but he is sure that the value will be between $50 \%$ and $80 \%$. Next, the simulation tool randomly generates for every attribute, a value in each one of those intervals. Then, to each tuple of attributes so defined will correspond a tuple of values for the variables resulting from the simulation. In this way, we generate a record for the database with the values of the attributes and the values obtained for the variables of the project that we want to analyze, that is to say, a specific scenario of the project. By repeating this process a certain number of times we can obtain a training file that serves as base for the mining process.

\section{APPLICATION OF GAR TO SDP DATABASES}

Before showing the results obtained by GAR, we will explain which are the adaptations we have to do in order that the tool should function according to the expectations of the experts. GAR searches for all the association rules that are in the database without taking into account the attributes that constitute the an- 
Table 1: Attributes used to generate the PDS database

\begin{tabular}{llcc}
\hline Abbreviation & Description & Unit & Initial value \\
\hline Inputs & & $\%$ & \\
ADMPPS & Average daily manpower per staff & days & 0.5 \\
ASIMDY & Average assimilation (of new personnel) delay & $\%$ & 20 \\
DEVPRT & Effort assumed needed for development & days & 0.85 \\
HIREDY & Hiring (of new personnel) delay & $\%$ & 0.4 \\
INUDST & Initial understaffing factor & $\%$ & 1.16 \\
MXSCDX & Maximum schedule completion date extension & days & 10 \\
TRNSDY & Time delay to transfer people out & $\%$ & 0.25 \\
TRPNHR & Number of trainers per new employee & $\%$ & 0.35 \\
UNDEST & Tasks underestimation fraction & & \\
Outputs & & technicians-days & 1111 \\
JBSZMD & Necessary effort to carry out the project & days & 320 \\
SCHCDT & Delivery time & errors/task & 0 \\
ANERPT & average number of errors per task & & \\
\hline
\end{tabular}

tecedent and the consequent. However, the project manager is who, in this case, decides which are the variables he wants to analyze. This means that such variables are the only ones that can appear in the consequent of the rules, while the rest of the attributes that the manager has chosen as input attributes will appear in the antecedent. What we have done, is to adjust the tool so it can only search for rules that have in the consequent the output variables.

The desire of the project managers is to find associations among the input parameters when the output variables are considered as good (low cost, acceptable delivery time, etc.). Nevertheless, a discrete class that shows when a project can be considered as good, bad or of other categories is not defined in the training file but a tuple of continuous values. Thus, the project manager must decide the intervals of the output variables values that make a project to be good. In this way, the manager defines the maximum percentages that can reach the value of a variable initially estimated in order to decide which are the values that make a scenario to be good or correct.

For example, supposing that the initial value estimated for the attribute SCHCDT (delivery time) is 320 days. If the manager decides that a project can be considered as good if does not overcome a $10 \%$ of the estimated initial value, the value of the variable SCHCDT must always been less than 352 days. In the same way, if the initial value estimated for the attribute JBSZMD (necessary effort) is 1111 technicians-day and the maximum percentage allowed is $20 \%$, the projects considered as good must not overcome 1333 technicians-day.

These maximum percentages has the name of cuts, since they are the limits allowed by the manager to consider that the scenario has been correct. So, and in order to make easy the search process, we have also adjust the tool to show only the associations rules with the values of the consequent moving within the ranges considered as good.

Departing from these accounts, GAR tool gives the project manager a set of association rules with a certain level of support and confidence that relates value intervals of the input parameters to value intervals of the output variables when these move within a certain range.

\section{ASSOCIATION RULES OBTAINED BY GAR}

In this section we describe the databases generated by the simulator and the goals we pretend to achieve. Next, we show the rules obtained by the tool in two databases using different management policies.

Once we have obtained the association rules (management rules), the project manager will be who decide which rule or rules are the easiest to be applied depending on the specific project and the software organization he is working on. He will also be who, before beginning the development and once the attributes that appear in the obtained management rules are analyzed, decide which attributes can be moved to keep them within the values that appear in the rules and which not, in order to optimize the results of the variables or set of variables of his concern. In any case, he will also know that if he does not keep within the obtained values he will not have guaranteed the optimization of the results.

In this study we have focused on determining over the variables of an already finished project, the influence that the management policies of hiring, adequacy and personnel dismissal have, when there is also initial restrictions on delivery time. That is to say, we want to analyze the influence that the personnel management policies have on a project with strong initial restrictions on delivery time, on the out- 
Table 2: Intervals estimated for the attributes

\begin{tabular}{lcc}
\hline Parameter & CRCCRT & GENERAL \\
\hline ADMPPS & - & {$[0.5,1.0]$} \\
ASIMDY & {$[5,15]$} & {$[10,120]$} \\
DEVPRT & - & {$[0.5,0.95]$} \\
HIREDY & {$[5,10]$} & {$[5,40]$} \\
INUDST & - & {$[0.2,1.0]$} \\
MXSCDX & {$[1,1.2]$} & - \\
TRNSDY & {$[5,10]$} & - \\
TRPNHR & - & {$[0.05,0.4]$} \\
UNDEST & - & {$[0.05,0.6]$} \\
\hline
\end{tabular}

put variables: necessary effort to carry out a project (technicians-day), development time (days) and quality (average number of mistakes by task). Specifically, the attributes whose values we want to know are: average delay in hiring, average delay in the adaptation of new technicians, average delay to carry out a dismissal and the maximum percentage of delay allowed in delivery time.

\subsection{Description of the Databases}

To carry out a study with GAR, we have simulated an already finished project, whose initial values are in table 1, following two strategies and, therefore, we have generated two databases. The first one, which we will call CRCCRT, has been generated establishing a fast hiring policy with initial restrictions on delivery time ${ }^{5}$.

The second one, which we will call GENERAL, has been generated with a less restrictive policy, in such a way that we have included in the simulation a greater number of attributes to be estimated and we have expanded the ranges of the values that indicate that a project is good for the output variables.

In table 1, we show the input attributes and the output variables used in the simulation together with a brief description, the unit in which they are measured and the initial value. In table 2, we show the intervals used for each one of the attributes with some level of uncertainty, for both databases. As can be seen, in GENERAL the intervals for attributes ASIMDY and HIREDY form all the possible range in the development organization. This is so, since with GENERAL database we pretend to analyze the influence that a greater number of attributes have on the project. In table 3 we show the cut values, that is, the values considered as good for both databases, CRCCRT and GENERAL, by the project manager. We have defined a cut for CRCCRT and two cuts or percent-

\footnotetext{
${ }^{5}$ Fast hiring implies that hiring (HIREDY), dismissal (TRNSDY) and adaptation of new technicians (ASIMDY) have to be realized quickly (MXSCDX), that is to say, in a short period of time (see table 2)
}

ages for GENERAL, which generate certain values for the output variables. These cuts are only applied to some of the output variables (except for cut 1 in GENERAL, which is applied to the three variables). Each one of these cuts establishes different correct scenarios. These cut values indicate the goals we pretend to cover. For example, the cut in CRCCRT has as goal to obtain management rules that should permit to maintain the delivery time and the quality of the project below the indicated values, independently of the value obtained by the effort necessary to carry out the project.

Together with the cut value, we show the maximum percentage, departing from the initial value estimated by the manager of the project, that must not overcome that variable in order to realise a project that adjusts to the initial estimations. For example, in the cut of CRCCRT we consider good values for delivery time those included between the initial estimation (320 days) and a permitted margin of $10 \%$ over such estimation (352 days), independently of the value obtained for the cost of the project. In this same table, we also offer information about the number of cases that have been categorised as acceptable. As can be seen, and as could be supposed from the very beginning, the number of cases decreases as the restrictions on the project increase. We can deduce, that too many restrictions could cause a low probability of carrying out an acceptable development project or even, that the probabilities were non-existent. For example, the fact of imposing a restriction on effort (JBSZMD) for the database CRCCRT, would provoke that the number of cases were practically nothing.

\subsection{Analysis of CRCCRT}

The database CRCCRT has been generated by imposing, in the simulation, restrictions on the attributes related to personnel hiring, making it to be fast, and, besides, by imposing strong initial restrictions on delivery time.

With the strategy followed in the generation of this database, we pretend to know the value of the attributes related to personnel management that permit to obtain good results for delivery time and to maintain acceptable levels in the project quality, independently of the value obtained for the necessary effort to carry out the project.

\subsubsection{Time and Quality}

This cut induces a set of rules on the input attributes for this database, only fulfilling restrictions on delivery time (SCHCDT) and quality (ANERPT), according to the cuts established in table 3.

The association rules discovered, where the consequent is formed by the intervals of the variables 
Table 3: Values and percentages of the output variables considered as good by the project manager

\begin{tabular}{lcccc}
\hline Cuts & JBSZMD & SCHCDT & ANERPT & \#cases \\
\hline CRCCRT & & $\leq 352(10 \%)$ & $\leq 0.45(12.5 \%)$ & 45 \\
Cut & - & $\leq 20 \%)$ & \\
GENERAL & & & & \\
Cut 1 & $\leq 2092(90 \%)$ & $\leq 387(20 \%)$ & $\leq .40(0 \%)$ & 48 \\
Cut 2 & $\leq 2092(90 \%)$ & $\leq 387(20 \%)$ & - & 221 \\
\hline
\end{tabular}

SCHDT [320,352] and ANERPT [0,0.45], that is to say, the management rules that would have permitted to obtain good results simultaneously for delivery time and project quality, are the following ones:

- $R_{1}:$ ASIMDY $\in[12.4,14.6]$ and HIREDY $\in[6.7,9.3]$ and MXSCDX $\in[1.10,1.18]$

( $\sup =7 \%$, conf $=91.3 \%$, \#cases=21)

- $R_{2}:$ ASIMDY $\in[12.5,14.4]$ and HIREDY $\in[7.6,9.9]$ and MXSCDX $\in[1.11,1.19]$ and TRNSDY $\in[5,7]$ (sup=3\%, conf $=100 \%$, \#cases=10)
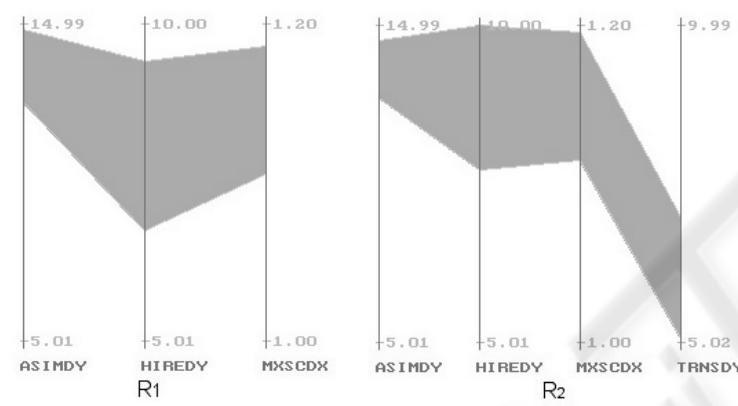

Figure 1: Rules obtained by GAR in CRCCRT

The quantitative interpretation we can withdraw from the rules discovered by GAR is the following: the support indicates the number of cases (percentage) where the conjunction of values of the rules is produced. The confidence shows the percentage of cases that fulfil such conjunction and besides, fulfil the values of the consequent. Together with this information, we show the number of cases that fulfil the rule in a correct way (\#covered cases) and the number of cases that also belong to other rules (\#rc). This interpretation will be applicable to the rest of the examples that are shown next.

In this particular case, rule $R_{1}$ shows that $7 \%$ of the cases (21) fulfil the conjunction of values ASIMDY $\in[12.4,14.6]$, HIREDY $\in[6.7,9.3]$ and MXSCDX $\in$ $[1.10,1.18]$ with the correct consequent, that is to say, with the values of the variables within the range established by the manager. The confidence value of $91.3 \%$ shows that in the database there are 23 cases that fulfil the antecedent, but that 2 of them do not fulfil the consequent. Rule $R_{2}$ shows that $3 \%$ of the cases (10) fulfil the antecedent and the consequent.
Besides, a $100 \%$ of confidence for this rule, means that all the cases (10) that fulfil the antecedent, also fulfil the consequent. We also want to show that 7 of the cases covered by this rule are also covered by other rules (\#rc=7).

In figure 1 we show the graphic representation of these rules. We can check that the average delay in the incorporation of new technicians to the project and their adequacy are attributes that appear in all the obtained rules. In all of them, such attributes take values from medium to high within the interval defined as fast personnel management. Facing the results obtained, $R_{1}$ would have been the easiest to be applied since we would have only to modify the values of ASIMDY and HIREDY.

\subsection{Analysis of GENERAL}

The database GENERAL has been generated with a less restrictive policy than the previous one, in such a way that we have expanded both the number of attributes to estimate and the cut percentage that establishes when the development of a project is good.

Given that the real values of this project for time and effort were of 387 days and 2092 technicians-day respectively, what we are searching for in this analysis, are the management rules that would have improved the final values of this project, keeping, at the same time, the quality within acceptable levels, as it is shown in table 3 . We can check again, the same than in the previous section, that if we limit the goals, that is to say, if we only want to obtain, in a global way, good results for time and effort, independently of the values obtained for the project quality, the number of suitable scenarios considerably increases (goes from 48 to 221 ).

\subsubsection{Cut 1: Time, Effort and Quality}

The first cut for this database is the only one that establishes restrictions on the three output variables. Therefore, the rules that are induced on the attributes must fulfil the three restrictions simultaneously, according to the cuts shown in table 3 .

The association rules discovered, where the consequent is formed by the intervals of the variables JB$\mathrm{SZMD} \in[1111,2092]$ and SCHCDT $\in[320,387]$ and 
ANERPT $\in[0,0.40]$, that is, the management rules that would have permitted to obtain, simultaneously, good results for delivery time, effort and project quality, are the following ones:
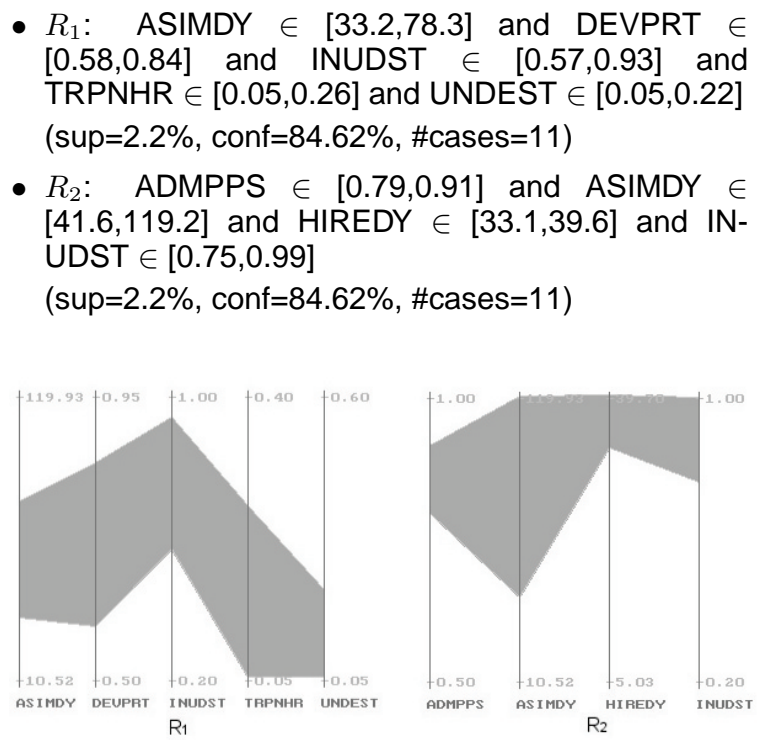

Figure 2: Rules obtained by GAR in GENERAL with cut 1 .

Figure 2 shows the graphic representation of the discovered rules. In this case, $R_{1}$ is the chosen rule for being realizing a post mortem analysis of the project. This rule, although involves 5 attributes (one more than rule $R_{2}$ ), is the one that is closer to the estimations realized for this project. That is to say, we would have improved the obtained results if we had improved the initial estimations on the size of the project (UNDEST), if we had increased the average delay of adequacy of the new technicians (ASIMDY) and if we had increased the number of technicians at the beginning of the project (INUDST). Rule $R_{2}$ would imply the modification of the four attributes since, the estimated values for each one of them are not in the range of the values indicated in the rule.

In figure 3 we compare the project nominal evolution with the evolution we would have obtained if we had applied the rule $R_{1}$. The value obtained for delivery time was 383, while for effort was 1647 technicians-day.

\subsubsection{Cut 2: Time and Effort}

As the goals of the project are less ambitious, we can check in this case that a larger number of possible scenarios have been found and that the attributes that we have to control in order to fulfil the goals of the project are smaller.

The association rules discovered, where the consequent is formed by the intervals of the variables

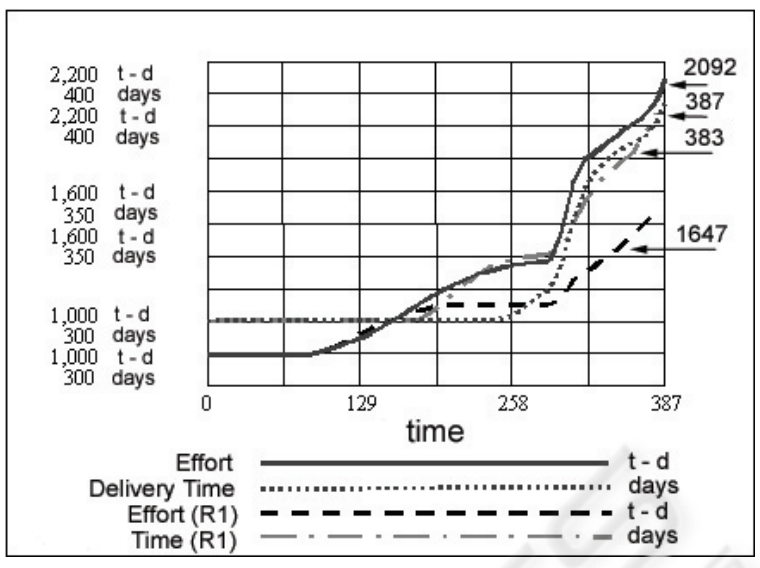

Figure 3: Comparison between the nominal simulation with the one of the project for R1.

JBSZMD $\in[1111,2092]$ and SCHCDT $\in$ [320,387], that is, the management rules that would have permitted to obtain, simultaneously, good results for delivery time, and effort, are the following ones:

- $R_{1}:$ INUDST $\in[0.64,0.99]$ and UNDEST $\in$ [0.05,0.48]

( sup=32\%, conf=93.02\%, \#covered=160)

- $R_{2}$ : INUDST $\in[0.59,0.86]$ and UNDEST $\in$ [0.25,0.44]

( $\sup =11 \%$, conf=100\%, \#covered=54)
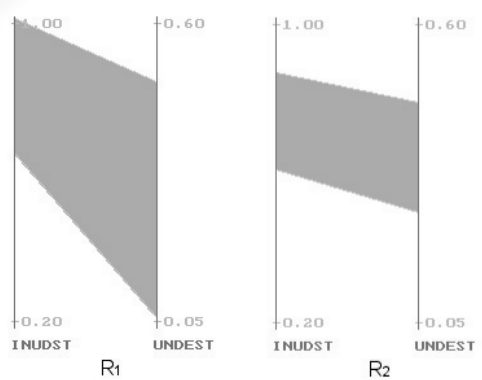

Figure 4: Rules obtained by GAR in GENERAL with cut 2 .

In figure 4 we show the graphic representation of the rules discovered by GAR. Following the criteria proposed by the project manager, to apply these rules, we only would have to modify an attribute in them, since, the underestimation of the size of the project is maintained within the levels initially established and we would only have to modify the value of the percentage of technicians that begin the project. The selection between $R_{1}$ and $R_{2}$, will depend exclusively on the project manager, as we have said before, according to his knowledge on the possibility of maintaining the implied attributes within the intervals provided by the rules. 
In all the rules obtained, both in cut 1 and in cut 2 , we can notice the importance that the percentage of technicians that begin the project has with regard to the whole estimated. In all of them, this attribute takes high values within the interval. This attribute is of great importance in the development process, since the greater its value is, the less the necessity of including new technicians to the project once this has begun will be. This implies a greater stability in the working team, less time spent in training, etc.

\section{CONCLUSIONS}

Software project simulators permit to model the complex behaviour of the software development process and to generate different scenarios for a same project. One of the most important advantages of these simulators lies in the fact that we can "test", without any cost, the effect that the application or not of different management policies will have on the project. Although, one of the greatest disadvantages is that we must estimate a great number of attributes (more than 60 in some of them) to know the project evolution. This means that the project manager has the difficult task of estimating not only the most suitable value for each one of them but, also, the optimum combination of such attributes. This obstacle has been solved in recent works by means of the use of machine learning techniques and by databases generated by a project simulator (Ramos et al., 2001)(Aguilar et al., 2001). The obtained management rules are going to permit us to know within which intervals the attributes about which we really have any uncertainty can move. Thus, we can decrease the uncertainty we have about certain management policies when we are estimating the project and check, in already finished projects, the accuracy of the realized assessments.

In this paper, we have presented GAR as a machine learning technique to generate management rules that help to the decision-taking in the SDP.

Finally, we want to indicate that, the application of the machine learning techniques to real historical bases, would permit to obtain management rules based on the experience of the work carried out to date by the development organization. So, if this happens, we can say that the experience acquire by a development organization will be useful to the managers to estimate and manage future projects and will not be exclusive of only one person.

\section{REFERENCES}

Abdel-Hamid, T. and Madnick, S. (1991). Software Project

Dynamics: an integrated approach. Prentice-Hall.
Agrawal, R., Imielinski, T., and Swami, A. (1993). Mining association rules between sets of items in large databases. In Proceedings of the 1993 ACM SIGMOD International Conference on Management of Data, pages 207-216, Washington, D.C.

Aguilar, J., Ramos, I., Riquelme, J., and Toro, M. (2001). An evolutionary approach to estimating software development projects. Information and Software Technology, 43(14):875-882.

Chichakly, K. (1993). The bifocal vantage point: managing software projects from a systems thinking perspective. American Programmer, pages 18-25.

Ramos, I., Aroba, J., and Riquelme, J. (2001). Improvements in the decision making in software projects. In Third International Conference on Enterprise Information Systems ICEIS'2001, pages 431-436, Setbal, Portugal. Proceedings, Vol. 1, P. Miranda et al (Eds). 